\title{
Experimental investigation of a 2d impinging jet on a liquid surface
}

\author{
R. Berger ${ }^{1,2}$, S. Depardon ${ }^{1}$, P. Rambaud ${ }^{2}$ \& J. M. Buchlin ${ }^{2}$ \\ ${ }^{I}$ Division of Research and Automotive Innovation, \\ PSA Peugeot Citroën, France \\ ${ }^{2}$ Environmental and Applied Fluid Dynamics Department, \\ von Karman Institute, Belgium
}

\begin{abstract}
An experimental investigation of impinging $2 \mathrm{~d}$ planar air jet on a water surface is performed by means of Particle Image Velocimetry (PIV). The difficulty encountered to measure the two dimensional velocity and turbulence fields close to the interface requires a special treatment of the PIV data obtained in the air and water sides. The resulting technique enables the localisation of the moving interface, the use of the inter-correlation and the calculation of statistics in both phases. The results show that the proposed PIV interface detection technique is in good agreement with the LeDaR detection technique developed at the von Karman Institute. The analysis of the air and water velocity field close to the interface highlights the topological differences between dimpling and incipient splashing configurations. The results obtained in this study will serve to model later turbulence transfer in impinging jet configuration.
\end{abstract}

Keywords: turbulence, $2 d$ impinging jet, interface, PIV-LeDaR.

\section{Introduction}

Gas jets impinging a liquid surface are encountered in a broad swathe of engineering processes related to automotive, metallurgical, chemical industries and safety systems. The gas jet impingement causes a depression at the liquid surface.

The development of the lateral gas flow from the impact point induces water motion and wave formation. However, the physical interaction between a gas (air) and a liquid (water) involves a number of complex phenomena occurring in 
both phases at the vicinity of the interface. The momentum transfer from the air to the water produces waves on the surface of the liquid and involves underwater flow and turbulence. The study of the flow structure above and below the interface is a very challenging task, especially in the presence of waves. The airflow structure above water surface has been studied for 50 years but the contribution of the near surface turbulence to momentum exchange between the two fluids is not well quantified and its modelling often in default. This lack of knowledge comes mainly from the difficulties to measure with accuracy physical quantities in the near interface region. In carrying a study of wind-wave flumes in oceanography, Shaikh and Siddiqui [1] show that PIV is a reliable optical nonintrusive measurement technique for investigating interface problem.

However, it is also known that PIV measurements are very sensitive to the quality of the image [2], so that the moving interface needs to be accurately located. Another limitation in the previous near interface PIV studies is the lack of simultaneous measurements. Indeed, lots of measurements and analysis are done either in the liquid phase [3,4] or in the gas phase [1], but very none are addressed to simultaneous measurements in both phases.

The present study is focused on the development of a PIV technique to be applied both in air and water above and below an interface in the case of a planar $2 \mathrm{~d}$ impinging jet. It is a pioneer study, which reports an analysis of the structure of the flow in both phases in the case of an impinging jet configuration and which yields a better understanding of the related mechanisms.

\section{Experimental setup}

The experiments are conducted on a dedicated setup designed and built at the von Karman Institute for fluid dynamics in Rhode-Saint-Genèse, Belgium (Figure 1). The facility is composed of a $1 \mathrm{~m}$ long, $30 \mathrm{~cm}$ wide and $15 \mathrm{~cm}$ high transparent Plexiglas tank that allows optical measurements. In this tank, a removable double floor is used to set the water depth around $15 \mathrm{~mm}$ and the distance with the nozzle outlet close to $30 \mathrm{~mm}$.

The $2 \mathrm{~d}$ planar jet is created by a nozzle with an outlet slot of $5 \mathrm{~mm}$. The nozzle is composed of a "stagnation" chamber and a convergent section. The nozzle is fed a 10 bars compressed air system through a pressure regulator that sets the flow rate, which is determined from the measurement of pressure inside the stagnation chamber. The turbulence in the jet potential core is quantified by means of a hot wire and evaluated at $4 \%$.

The jet Reynolds number, Re, is based on the nozzle slit. In the present study the Re value of 2000 and 2700 are considered. They correspond to dimpling jet and incipient splashing jet, respectively, as defined by Molloy [5] (see Figure 2).

PIV [2], with parameters summarised in Figure 3, is used to measure $2 \mathrm{~d}$ instantaneous velocity fields in a plane perpendicular to the water surface along the centre line of the tank. The measurement chain is made of a $250 \mathrm{~mJ}$ two cavities pulsed Nd-Yag laser and a PCO 1280 x 864 pixels CCD camera with a $35 \mathrm{~mm}$ objective and their related acquisition system. The camera and the laser are synchronised at $3 \mathrm{~Hz}$ by means of a timing hub. The laser sheet is created and 


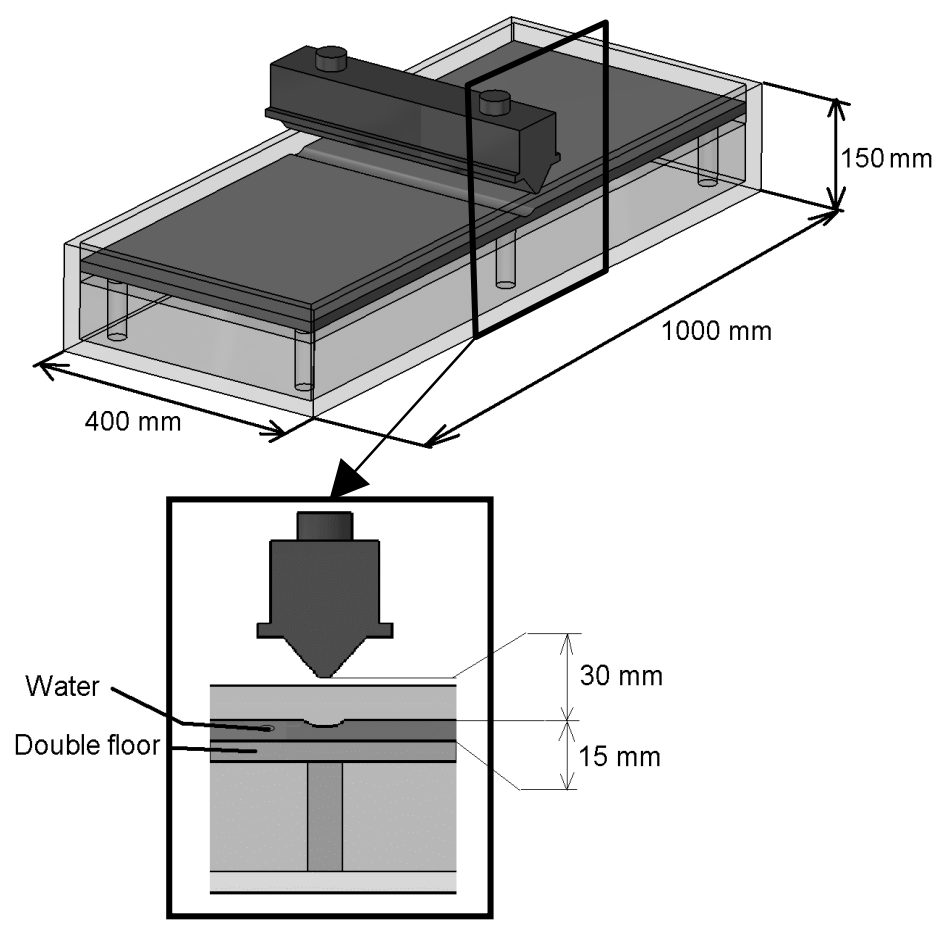

Figure 1: $\quad$ Schematic of the setup.

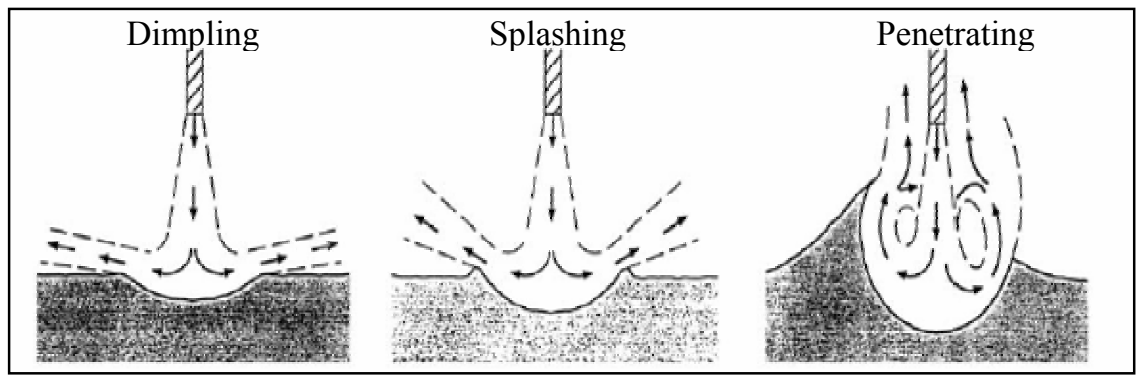

Figure 2: $\quad$ Impinging jet configurations - Molloy [5].

focused in the measurement plane with the use of a spherical and a cylindrical lens, which guide it to the measurement region by the side of the tank for the water PIV and by the bottom of the tank for the air PIV as shown in Figure 4. The instantaneous character of the PIV measurements allows the computation of detailed statistics of the flow field in terms of mean and fluctuating quantities. 


\begin{tabular}{|c|c|c|}
\cline { 2 - 3 } \multicolumn{1}{c|}{} & Air phase PIV & Water Phase PIV \\
\hline seeding particle & oil vapour & Vestosint \\
\hline Liquid & Water & Water - fluoreseine mixture \\
\hline Particle diameter & $\sim 1 \mu \mathrm{m}$ & $\sim 5 \mu \mathrm{m}$ \\
\hline field of view size & \multicolumn{2}{|c|}{$\sim 35 \mathrm{~mm} \times 25 \mathrm{~mm}$} \\
\hline separation time & $\sim 0.04 \mathrm{~ms}$ & $\sim 4 \mathrm{~ms}$ \\
\hline Processing software & \multicolumn{2}{c|}{ WIDIM [6] } \\
\hline initial window size & \multicolumn{2}{c|}{$26 \times 96$} \\
\hline refinement step & \multicolumn{2}{|c|}{$75 \%$} \\
\hline Overlapping & \multicolumn{2}{c|}{1 vector $/ \sim 0.17 \mathrm{~mm}$} \\
\hline final resolution & \multicolumn{2}{|c|}{}
\end{tabular}

Figure 3: $\quad$ PIV parameters.

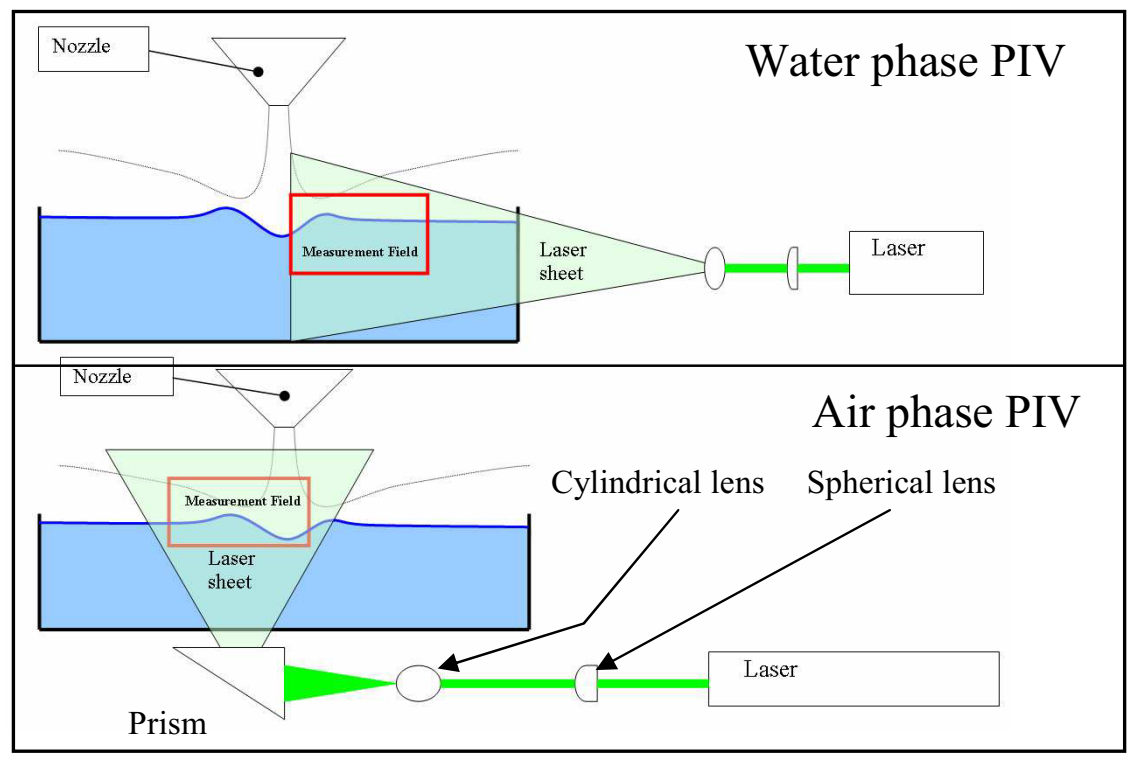

Figure 4: Laser arrangement overview.

In addition to PIV measurements, the LeDaR interface tracking technique developed at the VKI [7] is also applied to validate by comparison the interface detection from the PIV images. The LeDaR database is composed by 3000 images sampled at $2.5 \mathrm{kHz}$ rate by means of an $800 \times 600$ pixels high speed camera. The measurements allow also the detailed statistics of the interface deformation (mean and fluctuations), the dynamic analysis of the data.

\section{Interface detection}

In order to use WIDIM PIV algorithm developed at the VKI, a special treatment is used for each phase. The objective of these treatments is to provide images only in the medium (air or water) of interest. For both phases, the PIV images are 
used to detect the position of the interface. Once the interface is detected, a simple filtering is applied to blank the part of image that is not in the investigated phase. However, the procedure to determine the interface position is different depending on whether one uses air or water PIV data.

\subsection{LeDaR interface detection method}

Level Detection and Recording (LeDaR) algorithm is a reference measurement method for interface detection developed at the VKI. The principle and the performance of this technique are described by Planquart [8] and Bouchez [9]. The algorithm used is an improved version developed by Toth [10] for grey level sensor camera. The enhanced version used is based on the Maximum Forward Step Filter method that has already been shown [10] as the most robust detection method among the others available in LeDaR. An example of LeDaR interface detection is shown in Figure 5. Based on the accuracy of the algorithm and the spatial resolution, the resulting uncertainty of the interface location is estimated to be within $\pm 70 \mu \mathrm{m}$.

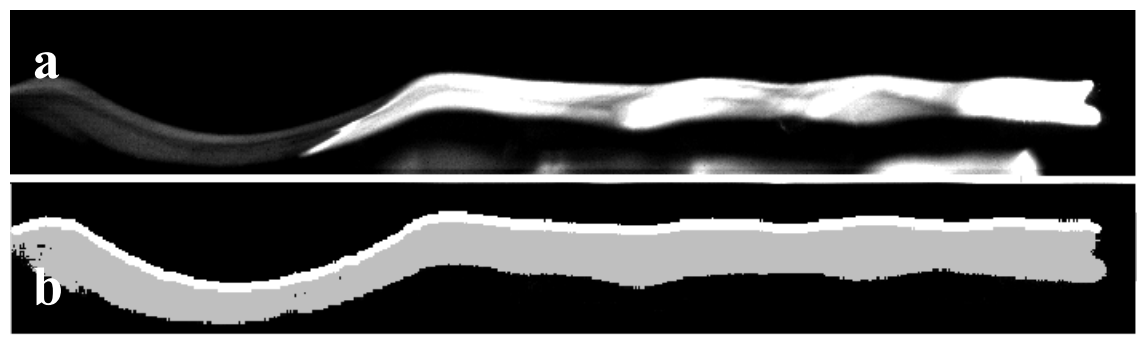

Figure 5: Example of LeDaR application: a - raw image, b - enhanced images with resulting interface (white line).

\subsection{Water phase interface detection}

From the water PIV raw images, the technique is relatively complex because the interface doesn't appear clearly due to the "Mirage effect" (Dias [11, Nogueira [12]). Therefore, an image texture separation has been developed and used (Figure 6).

The different steps of the detection procedure are successively:

- Grey level and Sobel filtering of the raw image to highlight the particle in the images. The use of the fluorescein is justified at this step for a better contrast and an efficient filtering of the image background and the "Mirage effect"

- Texture segmentation and closure. This step appears as the most critical because highly depend on the seeding density. It is required to be homogeneous between all the images for an automated process. The result is a binary image where the value " 0 " corresponds to the air phase and the value " 1 " to the water phase.

- Localisation of the binary jump for each pixels column, defined as the interface location 
- Spatial smoothing of the detected interface.

- Blanking of the above interface part in order to obtain the final images used for the cross-correlation process.

The process is applied to a set of 1000 images. The results show that small deviations of the interface appears due to strong light reflections on the interface by floating particles or low seeding density zones within less than $10 \%$ of the images. The difference between the computed interface and the real interface is manually checked on a set of 20 images randomly selected and for each one the maximal deviation recorded. The resulting uncertainty of the interface location is estimated to be within $\pm 180 \mu \mathrm{m}$. This ambiguity is mainly due to the closure and segmentation parameters, to the seeding density and the spatial resolution of the images.

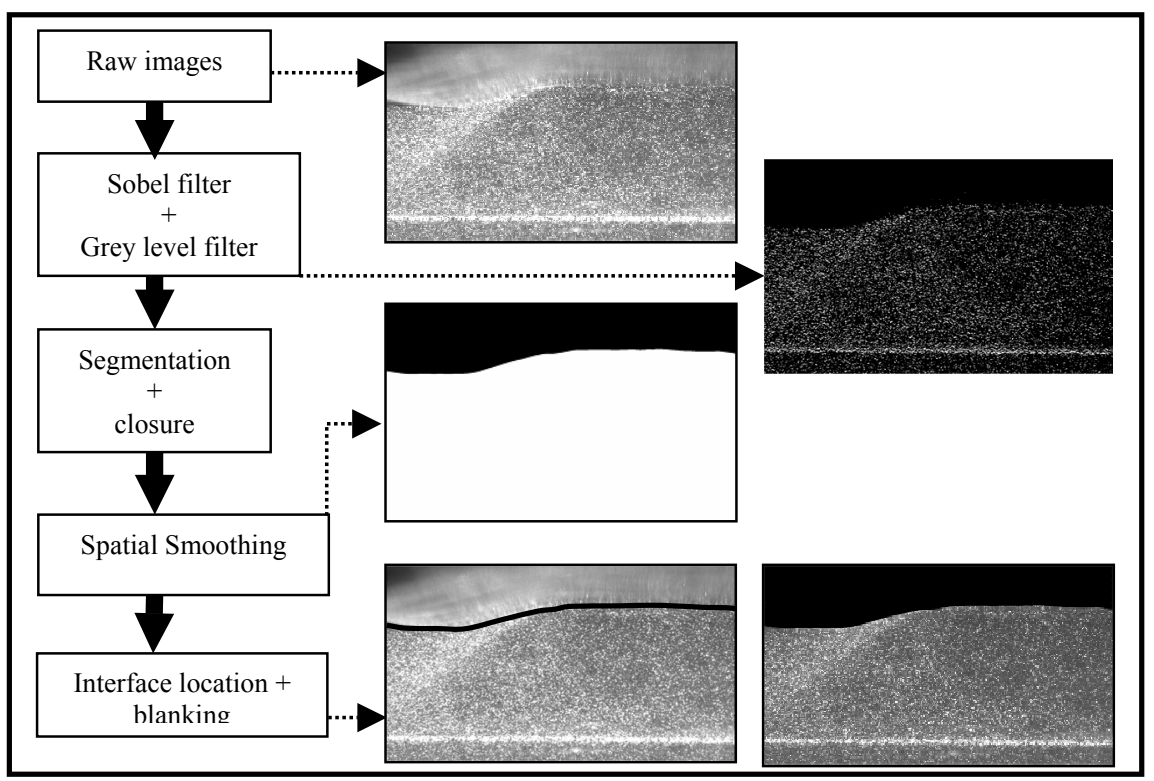

Figure 6: Water side PIV interface detection method illustration.

\subsection{Air phase interface detection}

The detection scheme from the air phase PIV is completely different. Indeed to avoid light saturation and to provide useful pictures for the interface detection, the setting respects the schematic drawn in Figure 7.

The way of detecting the interface in the air phase PIV image relies on the assumption that the deformed surface assumption has a $2 \mathrm{~d}$ character. Instead of detecting the interface in its real position (A in Figure 7), the interface is recorded as the image of the laser reflection footprint (B in Figure 7) on the wall of the double floor through the deformed interface ( $\mathrm{B}^{\prime}$ in Figure 7). This point is recorded as point $b$ on the camera picture. In addition we assume that the line of 
high intensity on the picture is the real interface deformation with a small shift that is very easy to determine manually. So applying the LeDaR algorithm [7] on the raw image and using a shift parameter we are able to accurately determine the position of the real interface (Figure 8). Applying the same accuracy evaluation than for the water phase, the interface is found to be localised within a tolerance of $\pm 120 \mu \mathrm{m}$. The determination from the air side is more accurate than from the water side thanks to the accuracy of the LeDaR algorithm.

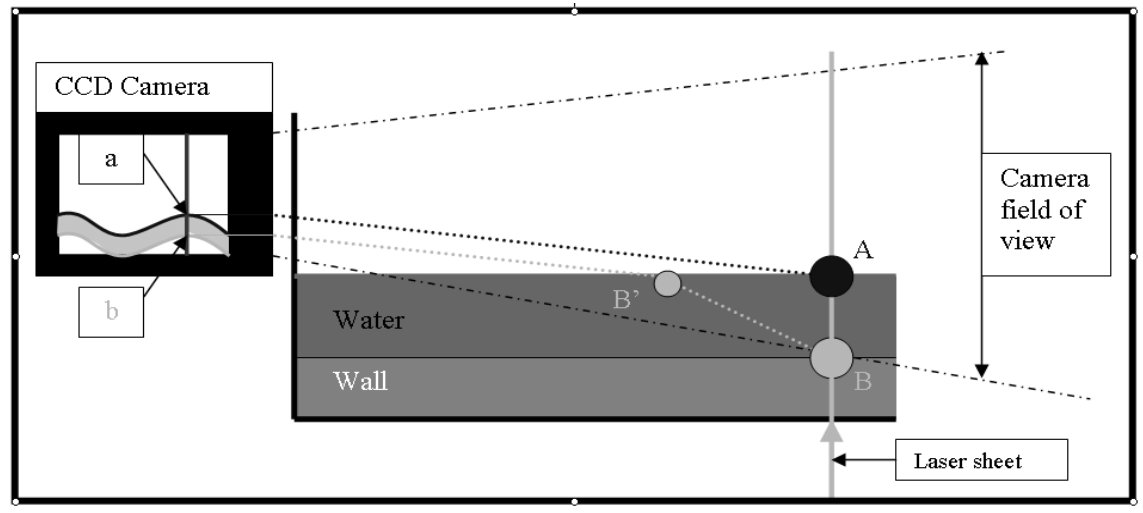

Figure 7: Detail of Air side PIV image.
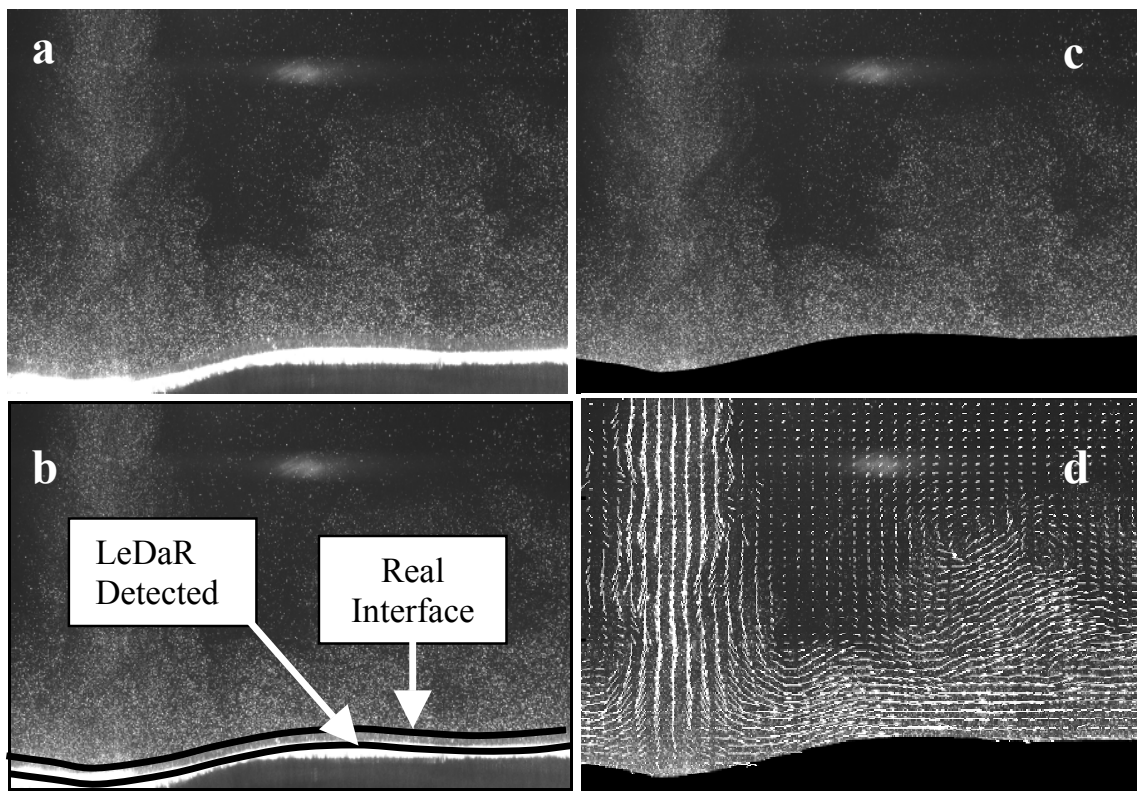

Figure 8: $\quad$ Air phase PIV based interface detection illustration: a - raw images, b - raw images with highlighted interface, $\mathrm{c}-$ blanked image, $\mathrm{d}$ - image with velocity vectors. 


\section{Results and discussion}

\subsection{Interface tracking scheme evaluation}

The PIV based interface localisation gives statistical information on the position of the interface that can be compared to the data obtained with a specific interface tracking measurement technique. The previous data extracted from the PIV are confronted with data obtained by the LeDaR technique. Figures 9 and 10 are typical examples. Figure 9 shows that good agreement is obtained between the interface tracking methods for the dimpling configuration of the impinging jet. The trough $(0<\mathrm{X} / \mathrm{b}<3)$ of the water surface described by Molloy [5] is retrieved by all the methods ("b" being the jet slot width).

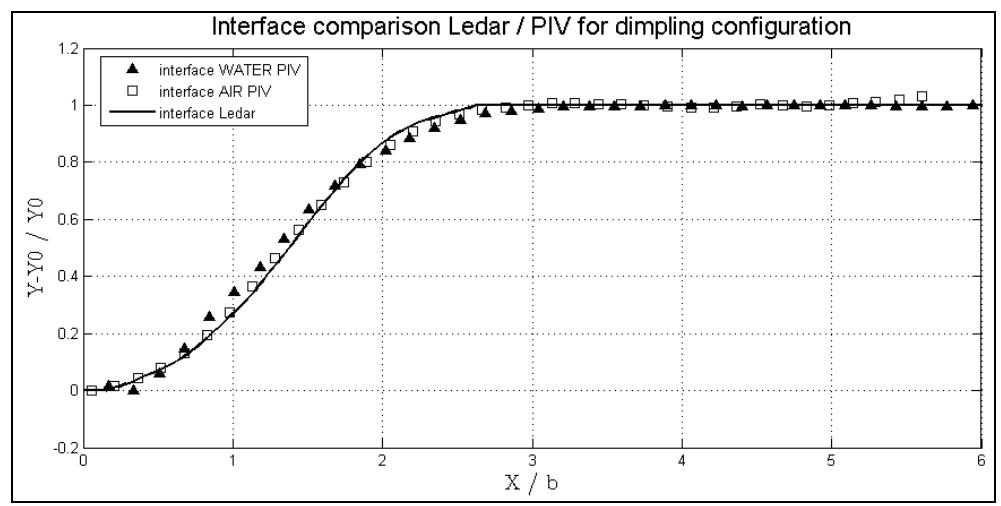

Figure 9: Interface location comparison for the dimpling configuration.

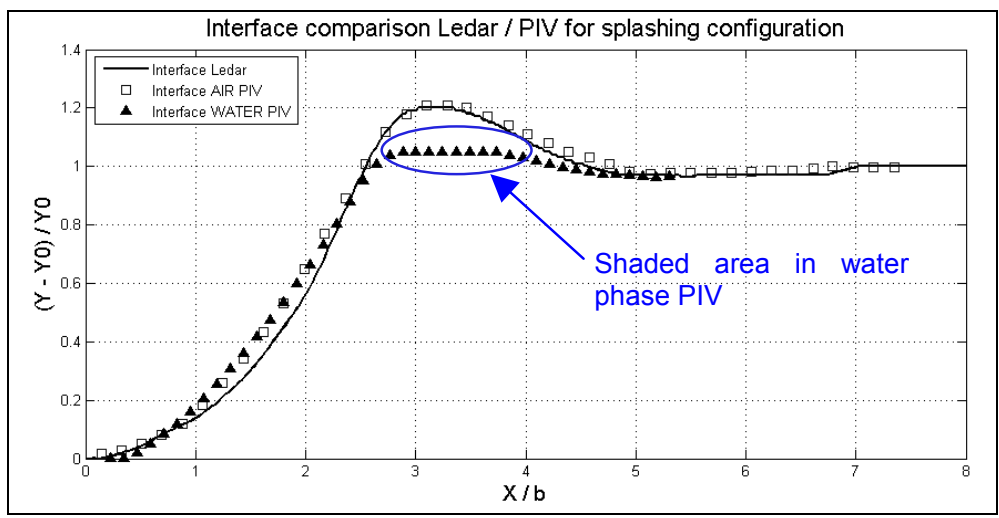

Figure 10: Interface location comparison for splashing configuration.

In the incipient splashing configuration (Figure 10), both techniques agree on the mean interface position in the depression region $(0<\mathrm{X} / \mathrm{b}<2.5)$ and the 
"interface flow" region $(\mathrm{X} / \mathrm{b}>4.5)$. However, the water PIV based interface position is underestimated in the region of the "lip" $(2.5<\mathrm{X} / \mathrm{b}<4.5)$. This inconsistency can be explained by lighting and seeding artefact. To obtain images respecting the needs of our interface detection scheme, the laser sheet is carried by the side of the tank. The consequence is the missing part upside the one detected is present in a shadow and low seeded region. The resolution of this problem is still under investigation. The consequence is a loss of PIV data in this region even though the technique gives satisfactory results in overall.

\subsection{PIV qualitative analysis}

* PIV provides a series of snapshots of the velocity field at fixed time interval. In a fixed Eulerian coordinate system, a time series of the velocity data at any spatial location between the top and the trough of the waves cannot be extracted. This limitation is mainly due to the fact that a given spatial location can either lie in the water or in the air. Hsu and Hsu [13] already show that time-averaged quantities do not exist in a useful form at a fixed position in the crest-trough region of a wavy interface. Two ways of computing the quantities are given:

- Conditional averaging. This method uses fixed spatial location and takes in account only the data that are in the investigated phase.

- Wave-following Eulerian averaging: in order to obtain time-averaged quantities especially for high amplitudes waves, Hsu and Hsu [13] as Shaikh and Siddiqui [1] use another system they show as more accurate and meaningful. They transformed the fixed Eulerian coordinate system into a wave-following Eulerian system. The transformation imposes that the new vertical origin of the coordinate system is located on the interface for each instantaneous field. The quantities are then arranged with reference to the water surface using a new vertical coordinate system $\zeta$, such that $\zeta=0$ is the location of the interface in the each field and the positive $\zeta$-axis is pointing upwards.

Since the first method could be sufficiently accurate for a smooth interface as observed in the dimpling condition, the issue could be more chaotic in the incipient splashing configuration where the surface becomes strongly wavy. That is why all the present study statistics are computed using the wave following coordinate system.

* Figure 11 shows the averaged velocity field on both phases for the two jet configurations. We can clearly distinguish the typical behaviour of each configuration with the lip presence $(\mathrm{X} / \mathrm{b} \sim 3)$. For both flow configurations the bulk rotating motion in the water phase, created by the water surface dragging [14] is captured. Compared to the dimpling configuration, the centre of the rotating motion in the incipient splashing configuration is located outside the field of view, because of higher velocity and wider water depression at the impingement.

* For both cases it is worthwhile focussing on the region close to the interface. The measurement resolution is not sufficient to check the velocity continuity 
across the interface due to the ene two order of magnitude difference between the velocities in both phases. Indeed, to be able to confirm continuity, we need to access the viscous sub-layer velocity in each phase as shown by Davis [15], who argues that the viscosity has a significant influence on the momentum transfer across the interface. That very small layer has not been resolved in the present study.

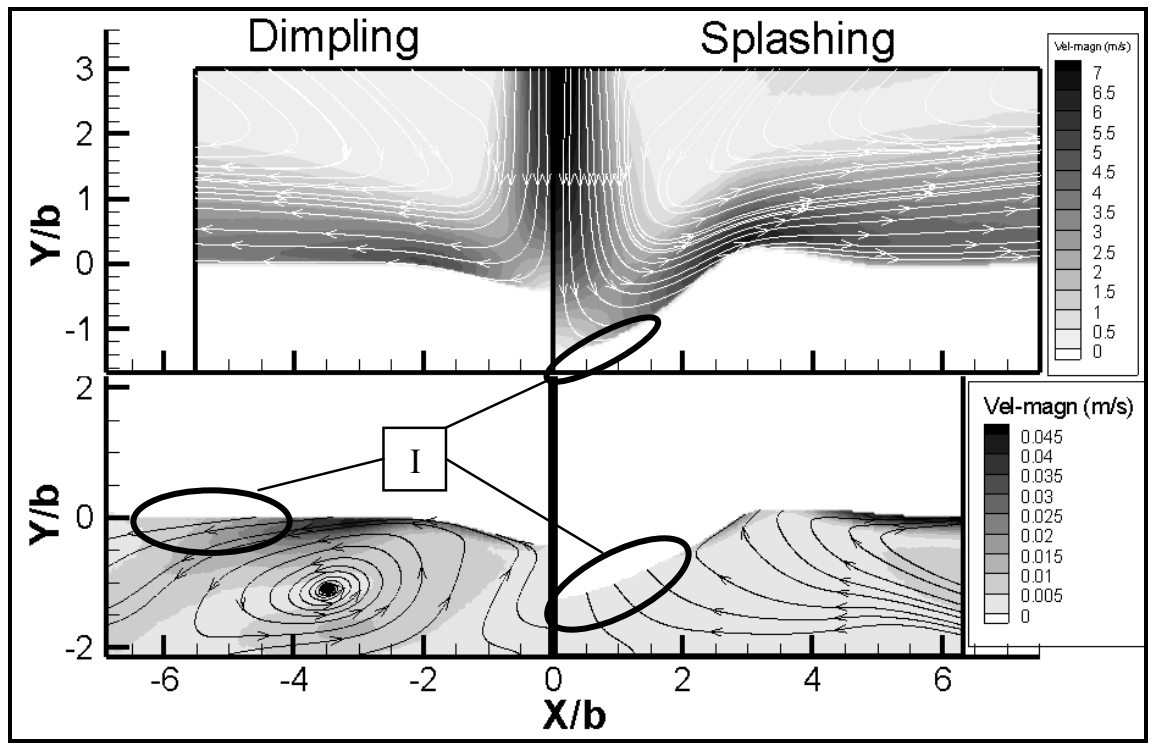

Figure 11: Water and air sides averaged velocity magnitude fields and streamlines $-\mathrm{X} / \mathrm{b}>0$ : splashing configuration $-\mathrm{X} / \mathrm{b}<0$ : dimpling configuration.

However, Figure 11 shows several regions (I) close to the interface where the streamlines cross the interface. The possible reasons of this wrong behaviour of streamlines are the lack of resolution close to the interface and the computation of the statistics (effect of the wave-following co-ordinate system, convergence). The resolution of these problems and the improvements of the results are under investigation.

\subsection{Momentum interaction between water and air phases}

* The air and water phenomena are clearly related as already mentioned by Komori et al. [16]. The air velocity magnitude reaches its maximum at the lip crest, at the same position that the maximum velocity in the water. The air velocity decrease in the "interface flow" region goes along with a water velocity decrease. It is obvious that the air flow is responsible for the water motion but the description of the fluid-to-fluid interaction at the interface remains incomplete. From the PIV averaged fields, two main zones are 
found be important for analysis of turbulence; the part at the crest of the lip, and the part at the trough of the lip named here as the "interface flow" region.

* As PIV measurements provide instantaneous velocity fields, they contain both mean and turbulent component of the velocity. As summarised by Shaikh and Siddiqui [1], the instantaneous velocity over a wavy surface is the sum of mean, turbulent and wave induced velocities. The conventional fluctuating velocity component $\mathrm{u}^{\prime}$ and $\mathrm{v}$ ' are computed using the Reynolds decomposition: $\mathrm{U}(\mathrm{t})=\mathrm{Um}+\mathrm{u}^{\prime}(\mathrm{t})$

$$
\mathrm{V}(\mathrm{t})=\mathrm{Vm}+\mathrm{v},
$$

where $\mathrm{Um}$ and $\mathrm{Vm}$ are the time averaged mean velocity components, $\mathrm{U}(\mathrm{t})$ and $\mathrm{V}(\mathrm{t})$ the instantaneous one. The Reynolds stresses are then computed as $-\overline{u^{\prime} \cdot V^{\prime}}$.

* As mentioned by Kato and Sano [18], this computed term represents the total Reynolds stress as u' and v' taken into account both wave-induced and turbulent velocities. Shaikh and Siddiqui [1], Friebel [19] make the observation that the positive Reynolds stress in the air phase just above an interface indicates that the transport of the turbulence is done towards the water surface. Using the same analogy, Shaikh and Siddiqui [1] observes that negative Reynolds stress in the air at the interface corresponds to the momentum flux transported from the fluctuating liquid surface to the gas phase. A similar discussion holds for water PIV analysis. In the present configuration, the Reynolds stress distribution is the same in the vicinity of the water surface for both dimpling and incipient splashing jet configurations. Furthermore, positive Reynolds stresses in the water come face to face with positive Reynolds stresses in the air, meaning that the turbulence in the air is transported through the surface to the water and vice versa for negative ones. Indeed, three important Reynolds stresses regions are denoted in the close vicinity of the surface.

As observed in Figure 13, upstream the lip crest (A), the Reynolds stresses remain positive, so the turbulence is transported toward the water. Then, at the lip crest (B), the Reynolds stresses becomes negative. It means that in this region, the water surface brings some turbulence to the air flow. And finally, downstream the crest (C), the Reynolds stress becomes positive, so the exchange of the turbulence is once again from the air to the water.

The mechanism of the turbulence transport in the $2 \mathrm{~d}$ impinging jet can now be described. The dynamic pressure of the jet imposes at the impingement a depression on the water surface. In this depression cavity, the turbulence of air flow associated with the pressure gradients impose to the liquid a motion. Moreover, the unsteadiness of the jet drives, by flapping on the surface, the interface into motion at the lip region. The continuous motion of the lip imposes the air flow to be continuously deviated at the crest, bringing to the air flow a new quantity of turbulence (wave-induced turbulence) highlighted by negative Reynolds stresses. Once the crest is reached, the generated turbulence is transported towards the liquid. The waves, because of their limited size, do not 


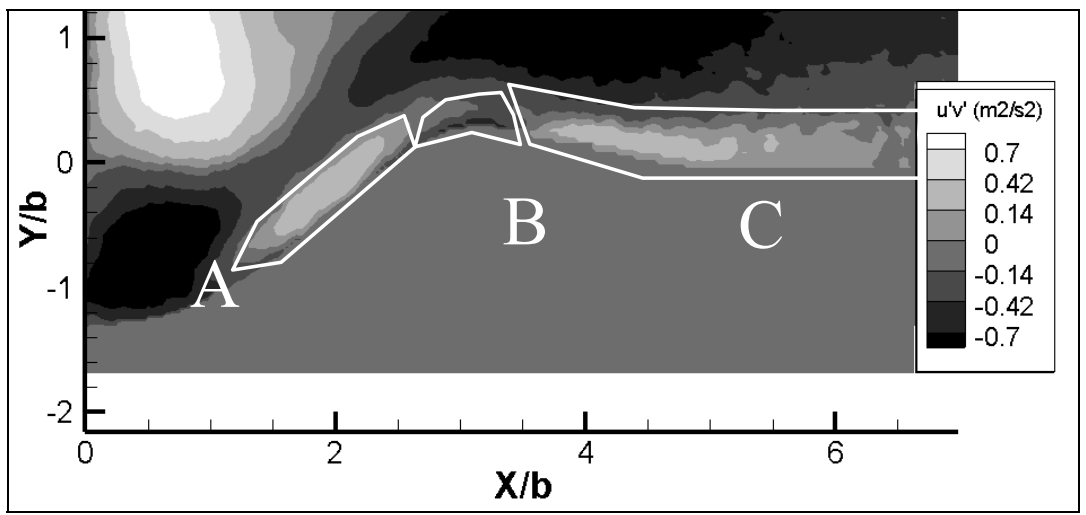

Figure 12: Reynolds stresses distribution along and in the vicinity of the interface in the air phase (splashing configuration).

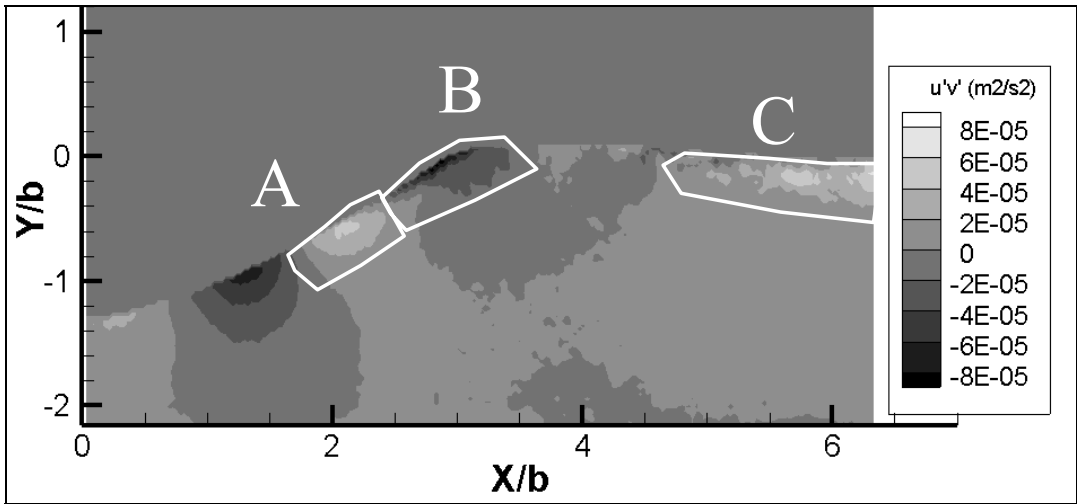

Figure 13: Reynolds stresses distribution along and in the vicinity of the interface in the water phase (splashing configuration).

act on the turbulence of the air flow and the Reynolds stresses remain positive. So it would be possible to correlate the unsteady phenomenon in air with unsteady motions of the interface. This issue is beyond the scope of the present study and will be a part of a forthcoming paper where a deeper analysis of the turbulence transport phenomena will be addressed.

These results demonstrate that the non-intrusive PIV technique can measure the velocity field above and below a water-air interface and properties of the field on both sides are closely linked by the turbulence properties of the field in the impinging jet configuration.

\section{Conclusion}

An experimental investigation of the flow field in the vicinity of an interface resulting from the impingement on a water layer of a $2 \mathrm{~d}$ dimpling and incipient 
splashing air jet is performed by the means of PIV. An innovative PIV technique is introduced to measure two dimensional velocity fields in both water and air phases. For each phase, a method is developed in order to detect the interface using only the raw PIV images. These 2 methods are confronted to a well-tried interface tracking method in order to be validated. The results show that the interface is determined with a good accuracy for each phase in both jet configurations. These methods give access to the turbulence properties of the flow of each phase in the very close vicinity of the interface. The analysis of the velocity and Reynolds stress fields highlights the turbulence transport mechanisms from the air jet towards the water flow. The interaction between the interface motion and the flow fields is also emphasized. The present study demonstrates that PIV is an efficient technique to measure with a good accuracy the phasic velocity fields in the near interface region of a gas jet impinging on a water surface.

The perspectives of this study are twofold; a deeper analysis of the turbulence transfer phenomena that take place in the interface region and subsequently the use of the database to validate CFD simulation such as VOF-LES.

\section{References}

[1] Shaikh N. and Siddiqui K., Air velocity measurements over the windsheared water surface using Particle Image Velocimetry, Ocean dynamics 58, 2008, pp65-79.

[2] Riethmuller M.L, Particle Image Velocimetry and associated techniques, 2000, VKI lecture series 2000 - 01.series 2nd revised edition 2007 - 01 .

[3] Pierson W.L., Measurement of surface velocities and shears at a wavy airwater interface using PIV, Exp in Fluids 23, 1997, pp427-437.

[4] Misra K.S., Thomas M., Kambhametu J.T., Estimation of complex air water interfaces from PIV images, Exp. in Fluids 40, 2006, pp764-775

[5] Molloy N.A., Impinging jet flow in a two-phase system: The basic flow pattern, J. Iron Steel Industry, October 1970, pp943-950.

[6] Scarano F., Particle Image Velocimetry, development and application, $\mathrm{PhD}$ thesis, universita di Napoli Frederico Secundo, Italy, 2000

[7] Toth B., Anthoine J., Riethmuller M.L., Mesure dynamiques de la deformée d'une surface libre, Congres francophone de techniques laser, 2006

[8] Planquart Ph., Real time optical detection and characterisation of water model free surface. IN proceedings of the $4^{\text {th }}$ European Continuous Casting Conference, 2002, Birmingham, UK

[9] Bouchez D., Zimmer L., Riethmuller M.L, Optical detection and characterization of interfaces, in Proceedings of the $9^{\text {th }}$ Symp. of flow visualizations, August 2000.

[10] Toth B., Two phase flow investigation in a cold gas solid rocket motor model through the study of the slag accumulation process, $\mathrm{PhD}$ thesis, 2008, von Karman Institute ULB. 
[11] Dias Pereira M., Bubble formation at a multiple orifice plate submerged in quiescent liquid, PhD Thesis, 1999, von Karman Institute - ULB

[12] Nogueira S., Flow around a single Taylor bubble rising through stagnant and co-current flowing Newtonian liquids

[13] Hsu C.T, Hsu E.Y., On the structure of turbulent flow over a progressive water wave: theory and experiments in a transformed, water-followed coordinate system. JFM 105, 1983, pp87-117

[14] Forrester S.E., Evans G.M., Computational modelling study of a plane gas jet impinging onto a liquid pool, Inter. Conf. in Mineral \& Metal Processing and Power Generation, 1997, pp313-320

[15] Davis R.E., on turbulent flow over a wavy boundary, JFM 42, 1970, pp721731

[16] Komori S., Nagaosa R., Murakami Y., Turbulence structure and mass transfer across a sheared air-water interface in wind-driven turbulence, JFM 249, 1993, pp161-183

[17] Hassain AKMF. Reynolds W.C., The mechanics of organized wave in turbulent shear flow, JFM 41, 1970, pp241-258.

[18] Kato H, Sano K, An experimental study of turbulent structure of wind over water waves. Rep Port Harb Res Inst 10, 1971, pp3-42

[19] Friebel HC., Air momentum flux measurements in the surf zone over braking waves, 2005, PhD thesis SIT Hoboken, New Jersey, USA 